\title{
Publication and patent analysis of European researchers in the field of production technology and manufacturing systems
}

\author{
Fiorenzo Franceschini • Domenico Maisano
}

Received: 10 January 2012/Published online: 17 February 2012

(C) Akadémiai Kiadó, Budapest, Hungary 2012

\begin{abstract}
This paper develops a structured comparison among a sample of European researchers in the field of Production Technology and Manufacturing Systems, on the basis of scientific publications and patents. Researchers are evaluated and compared by a variegated set of indicators concerning (1) the output of individual researchers and (2) that of groups of researchers from the same country. While not claiming to be exhaustive, the results of this preliminary study provide a rough indication of the publishing and patenting activity of researchers in the field of interest, identifying (dis)similarities between different countries. Of particular interest is a proposal for aggregating analysis results by means of maps based on publication and patent indicators. A large amount of empirical data are presented and discussed.
\end{abstract}

Keywords Research evaluation · Publications · Patents - Technology transfer · Production technology $\cdot$ Manufacturing systems

\section{Introduction}

Evaluating the performance of a research system is a complex and tricky activity wherein many aspects are involved. At the risk of oversimplifying, there generally are two main pathways of interaction between the research system and its environment (Shelton and Leydesdorff 2011):

- incoming resources, which are essential to feed the research system. They usually are human (e.g., staff) and/or economic-financial ones (e.g., public/private research funding);

F. Franceschini - D. Maisano (ه)

Politecnico di Torino, DISPEA (Department of Production Systems and Business Economics),

Corso Duca degli Abruzzi 24, 10129 Torino, Italy

e-mail: domenico.maisano@polito.it

F. Franceschini

e-mail: fiorenzo.franceschini@polito.it 
- research outputs, which can be divided in two main types: (1) scientific publications (e.g., journals papers, conference proceedings, book chapters, monographs, etc....), addressed to the scientific community, and (2) technology transfer applications (e.g., patents, university spin-offs, consulting services etc....), addressed to the industry and the whole socio-economic system.

Although the first type of research output (i.e., publications) is commonly recognised, the second (i.e., technology transfer applications, which constitute the so called third mission for university research systems) has been much discussed only in the last 10-15 years (Nagpaul and Roy 2003; Geuna and Nesta 2006). Nevertheless, technology transfer is particularly important for the applied scientific disciplines, since they are closely connected to industry and technology in general.

There is a double link between incoming resources and research outputs. While it seems reasonable that more resources are likely to produce more outputs (direct link), on the other hand, a significant part of the (future) resources may depend on the (past) outputs (reverse link). In this sense, there is no clear distinction between cause and effect. However, it can be said that generating good output is a necessary (but not sufficient) condition for a research system's life.

Indicators based on publications and patents — which are both objective and easily measurable quantities - are the most commonly used proxies for evaluating the previous two types of research outputs. In the literature, there are many cases in which these two typologies of indicators are used in combination. For instance, (Czarnitzki et al. 2007; Guan and He 2007; Calderini et al. 2009; Breschi and Catalini 2010) and many others. From most of these works, interesting results emerge about the potential correlation between intensity of research activity and patents.

The goal of this paper is to make a preliminary comparison among European researchers in the field of Production Technology and Manufacturing Systems, on the basis of the two analysis perspectives of publications and patents. While, in this specific field, some publication analyses have been recently presented in the literature (Franceschini and Maisano $2011 \mathrm{a}, \mathrm{b})$, there is lack of studies from the perspective of patents. This work should be useful for providing a rough indication on the different inclination of researchers to "classical" research, and technology transfer, investigating about possible interactions (Agrawal and Henderson 2002).

A homogeneous sample of researchers from several European countries was identified by referring to members of the CIRP (Collège International pour la Recherche en Productique, also known as International Academy for Production Engineering), one of the most important international associations of researchers in the discipline concerned (CIRP 2011). Specifically, we selected the researchers from the first nine European countries in terms of number of CIRP members. The choice of limiting the analysis to European researchers is aimed at making the comparison as homogeneous as possible, especially regarding patent analysis (Criscuolo and Verspagen 2008; Breschi and Catalini 2010).

Analysis is carried out by several indicators that are collected using the Scopus database. Input data are publications and patents, with corresponding citations. These data are used to construct other indicators so as to better depict the performance of researchers (Franceschini et al. 2007). Of particular interest is the intensive use of the Hirsch $(h)$ index and other $h$-based indicators, both at publication and patent level (Hirsch 2005; Guan and Gao 2009; Franceschini and Maisano 2011b).

While not claiming to be exhaustive and complete, the results of this preliminary study can be useful for: 
- providing a rough indication on the publishing and patenting activity of European researchers in the field of Production Technology and Manufacturing Systems, investigating possible relationships/interactions;

- identifying (dis)similarities between researchers from different countries, as regards their propensity to publish and patent (being aware that it can be strongly influenced by government policies or incentives).

\section{Methodology}

The same set of indicators is used for both the analysis perspectives of publications and patents. In case of potential ambiguity, when presenting the analysis results, these two categories of indicators will be distinguished by means of the superscript "(PUB)", for publication-related indicators, and “(PAT)", for patent-related indicators. Indicators can be in turn divided in: (1) indicators related to individual researchers and (2) indicators related to groups of researchers from the same country. They are summarised in Fig. 1 and described in detail in the following paragraphs.

All the indicators are calculated taking into account the publications/patents, and the corresponding citations, accumulated up to the moment of the analysis (February 2011).

Indicators for individual researchers

$P, C$ and $C P P-P$ is the total number of publications/patents and $C$ is the total number of citations received by the scientific publications/patents of a researcher. $P$ gives a quantitative information of the publishing/patenting activity. In case of publications, $C$ is informative of the total impact/diffusion of one researcher's scientific publications, while, in case of patents, $C$ roughly illustrates the overall knowledge flow generated by one researcher's patents. $C P P$ is the average number of citations per publication (i.e., $C / P$ ) and provides an indication of the average impact/diffusion. $C P P$ can be used to make

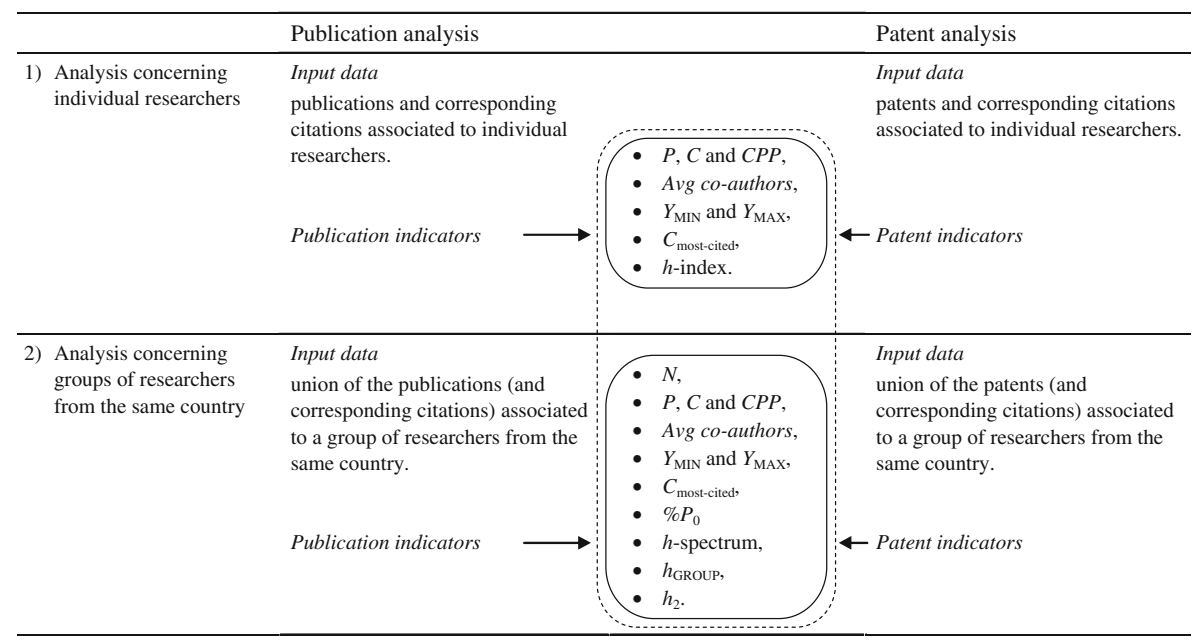

Fig. 1 Summary of the indicators in use. It can be noticed that the same indicators are used for both the publication and patent analyses 
comparisons between researchers, regardless of the fact that they have a different number of publications/patents.

$h$-Index - the $h$-index is a relatively recent but very popular indicator that synthetically aggregates two important aspects of the publication output: respectively impact/diffusionrepresented by the number of citations of a paper-and productivity-represented by the number of different papers (Hirsch 2005; Rousseau 2006; Egghe 2010; Franceschini and Maisano 2010a). In general, the larger $h$, the larger the diffusion and prestige of one author in the scientific community. The $h$-index can be also used to evaluate the technological importance and impact of one researcher's patent portfolio, simply considering the number of different patents and the number of citations of each patent (Guan and Gao 2009).

Avg co-authors is the average number of co-authors relating to publications/patents of one researcher. This indicator is symptomatic of the tendency towards co-authorship.

$Y_{M I N}$ and $Y_{M A X}$ are respectively the year relating to the oldest publication/patent and the year relating to the latest one. They provide a rough indication of the temporal extension of the publishing or patenting activity of a researcher.

$C_{\text {most-cited }}$ is the number of citations received by the most cited publication/patent of a researcher, representing the "jewel in the crown" in terms of impact/diffusion.

Indicators for groups of researchers from the same country

$P, C$ and $C P P, A v g$ co-authors, $Y_{M I N}, Y_{M A X}$ and $C_{\text {most-cited }}$ are exactly the same indicators seen in "Indicators for individual researchers" section. In this case, they are constructed considering the union of the publications/patents associated to a group of $(\boldsymbol{N})$ researchers from the same country.

$\% P_{O}$ is the percentage of researchers with no publications/patents. While it is (almost) impossible to find a researcher with no publications, on the other hand, it will be shown that many researchers do not have any patents.

$h$-Spectrum is defined as the distribution representing the $h$-values associated to a group of researchers. $h$-spectrum gives a "snapshot" of the population of a group (Franceschini and Maisano 2010b; Lazaridis 2010). We can distinguish between local $h$-spectra-i.e., those related to researchers of the same country - and a global $h$-spectrum, constructed considering the $h$-values of all the researchers at European level. Several indicators can be associated to the $h$-spectrum: the average $(\bar{h})$ and the median $(\tilde{h})$ as indicators of central tendency, the corresponding standard deviation $(s)$ and interquartile range (IQR) as indicators of dispersion.

$h_{\text {GROUP }}$ is the $h$-index of a group of researchers from the same country, that is to say the $h$-index of the union of the publications or patents associated to these researchers.

$h_{2}$ is the first successive $h$-index of a group of researchers. $h_{2}$ is defined in this way: a group has index $h_{2}$ if it has $h_{2}$ members with an $h$-index of at least $h_{2}$ (Schubert 2007). Subscript " 2 " denotes that this $h$-index (of level 2) represents the group's performance, on the basis of the individual researchers' $h$-indices (of level 1 , where subscript " 1 " is omitted). $h_{2}$ indicates the portion of members that "keep the show going" for one group of researchers, identifying the size of the most productive core of researchers.

\section{Data collection}

A first problem, which is only apparently trivial, is identifying a sample of homologous researchers, belonging to different European nations, but involved in similar research 
issues. For example, regarding public research institutions, the categorization of scientific fields may vary from country to country (Mattson et al. 2008).

The expedient used to select a homogeneous sample of researchers from several European countries, is to refer to members of CIRP, which is one of the major international associations of academic and non-academic researchers in the discipline concerned (CIRP 2011).

In this study, we selected about two-hundred total researchers, who are distributed among the following countries: Germany (62), United Kingdom (33), Italy (27), France (17), The Netherlands (17), Switzerland (13), Poland (10), Denmark (9) and Sweden (9).

For each of these researchers, publication/patent statistics were collected using the Scopus search engine. We chose this database for three main reasons: (1) in the field of Engineering Science, Scopus' coverage is superior to that of Web of Science (Bar-Ilan 2010); (2) Scopus is much more accurate than Google Scholar database (Labbé 2010); (3) Scopus integrates patent statistics from the major worldwide patent and organisations, i.e., EPO (European Patent Office), USPTO (United States Patent and Trademark Office), JPO (Japan Patent Office) and WIPO (World Intellectual Property Organization) (ScopusElsevier 2011).

Regarding publication statistics, Scopus makes it possible to quickly "isolate" researchers by their full first name(s) and affiliation. Nevertheless, seven researchers were excluded from the (publication) analysis, because of the risk of ambiguity.

Regarding patent statistics, data collection was much more difficult and time consuming. In fact, the Scopus patent database reports only the first name initials of a generic researcher, increasing the risk of homonymy. The number of researchers excluded from the patent analysis is doubled (14 researchers). Results associated to non-excluded researchers were examined carefully and cleaned.

The resulting samples of researchers used in the publication and patent analysis are summarized in Table 1, specifying how they are distributed among the different European countries.

Table 1 Country and staff number of the groups of researchers analysed

\begin{tabular}{lllll}
\hline Country & Group abbrev. & Staff number & & \\
\cline { 3 - 5 } & & Before exclusion & After exclusion & \\
\cline { 3 - 5 } & & & Publication analysis & Patent analysis \\
\hline Germany & DEU & 62 & 61 & 60 \\
United Kingdom & UK & 33 & 31 & 26 \\
Italy & ITA & 27 & 25 & 27 \\
France & FRA & 17 & 16 & 15 \\
The Netherlands & NED & 17 & 16 & 14 \\
Switzerland & CH & 13 & 13 & 13 \\
Poland & POL & 10 & 10 & 9 \\
Denmark & DEN & 9 & 9 & 9 \\
Sweden & SWE & 9 & 9 & 183 \\
& Total & 197 & 190 & 9 \\
\hline
\end{tabular}

In particular, we report the staff number before and after the exclusion of some researchers, for publication and patent analysis, respectively. Countries are sorted in descending order according to their staff number before exclusion 
After identifying the patents of each researcher, we determined the number of citations received. It may happen that sometimes, the same patent may have been deposited in more than one patent office. The (not very frequent) duplicate patents were identified quite easily (noting the title of the patent and the name of the inventors) and counted only once, whereas the corresponding citations were cumulated. We are aware that this citation "aggregation" could be questionable since the tendency toward citation may change from one patent office to the other (Criscuolo and Verspagen 2008). However, we believe that these "aggregated" citations give a reasonable indication of the overall impact/diffusion of a patent (Cheng et al. 2010).

\section{Analysis results}

Indicators (both at publication and patent level) concerning individual researchers are used to determine the indicators related to groups of researchers from the same country. Results are summarised in Table 2 and deeply discussed in the following paragraphs.

Figure 2 shows the (global) $h$-spectra related to the whole set of European researchers examined, respectively from the publication and patent perspective. As expected, distributions are right-skewed and the average $h$-index relating to publication analysis is significantly higher than that relating to patent analysis (see the last row of Table 2a, b) (Franceschini and Maisano 2010b).

Global $h$-spectra may represent a European reference for individual researchers within the area of interest. For example, a researcher with $h^{(\mathrm{PUB})}=3$ will fall on the 28th percentile. Analogous (local) $h$-spectra can be constructed for each of the nine groups of researchers from the same country.

Consistently with Lazaridis (2010), $\bar{h}$ is used as a synthetic indicator to perform quick evaluations and comparisons among the local $h$-spectra, even if-from a conceptual point of view-it would be more correct to use $\tilde{h}$. The reason is that $h$ is defined on an ordinal scale (Bornmann et al. 2008; Franceschini and Maisano 2010a).

Particularly interesting is the comparison between the researchers' $h^{(\mathrm{PUB})}$ and $h^{(\mathrm{PAT})}$ values. In general, the latter ones are very low (e.g., almost $70 \%$ of the researchers have $h^{(\mathrm{PAT})}=0$ ) for two main reasons: (1) patenting is a relatively rare event in the career of a researcher, as also confirmed by the very large portion of researchers with no patent $\left(\% P_{0}\right.$ (PAT), see Table 2); (2) only very few patents are cited heavily, also because it takes time for a patent to accumulate a large number of citations from later patents (Guan and Gao 2009). In this sense, for individual researchers, $h^{(\mathrm{PAT})}$ is significantly less effective than $h^{\text {(PUB) }}$, due to the lower discriminatory power.

$h_{\text {GROUP }}$ gives an indication of the impact of a group of researchers on the scientific community. As shown in Table 2, and confirmed by (Guan and Gao 2009), $h_{\text {GROUP }}^{(\mathrm{PAT})}$ does not suffer from the low discriminatory power of $h^{(\mathrm{PAT})}$, being based on a larger number of patents (and corresponding citations). Of course, large groups are favoured, since they generally have a larger number of publications and patents. For example, the group of German researchers (DEU) has the highest $h_{\text {GROUP }}$ value, both at publication and patent level. Thus, this indicator can not be used to make direct comparisons among groups with different size.

To make $h_{\text {GROUP }}$ values comparable and obtain an indication on the average performance of a group of researcher, complementary to the one provided by $\bar{h}$, a normalization has to be introduced. A possible way is to multiply the $h_{\text {GROUP }}$ values by the inverse of the 


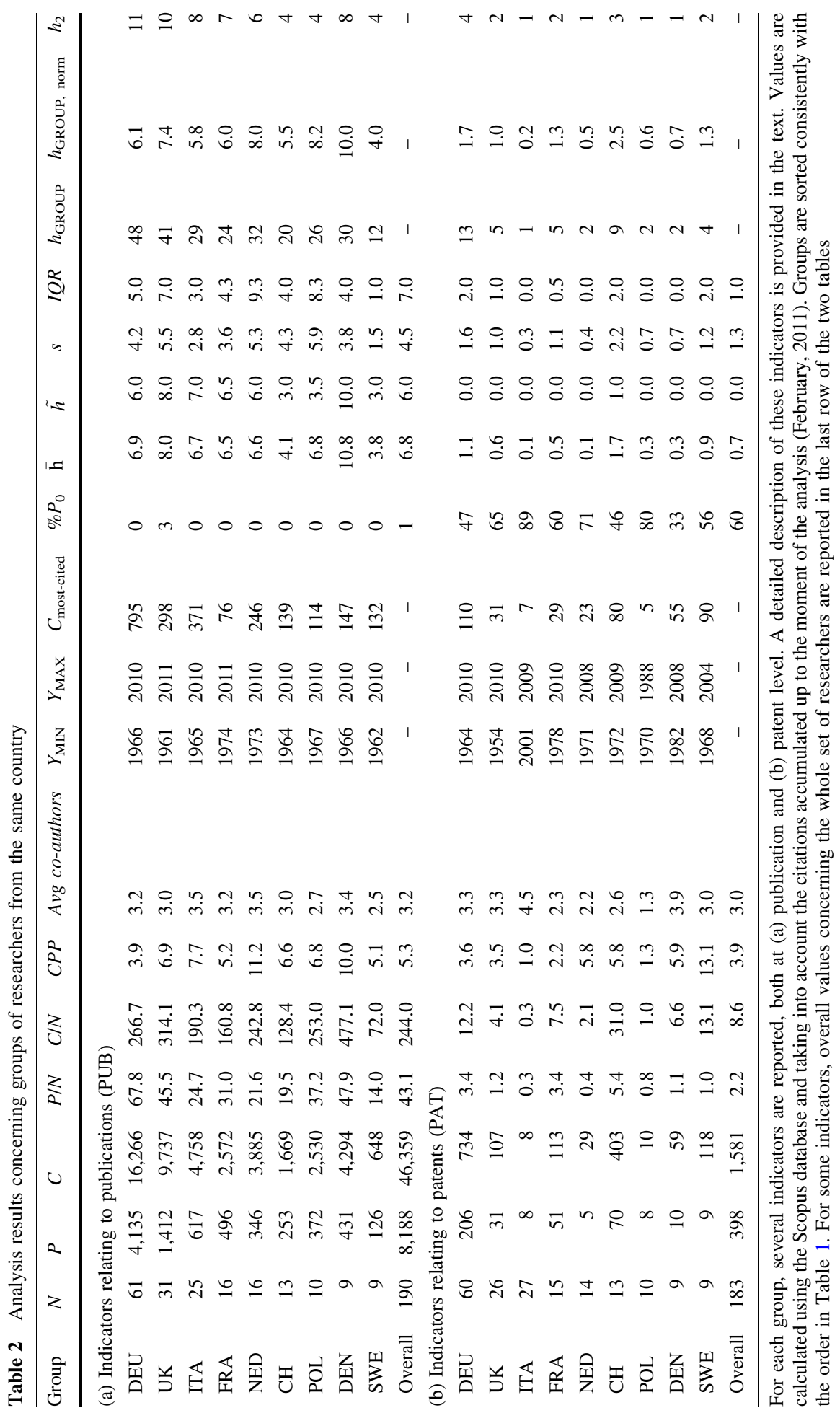


(a) Researchers' relative frequency versus $h^{(\mathrm{PUB})}$-index

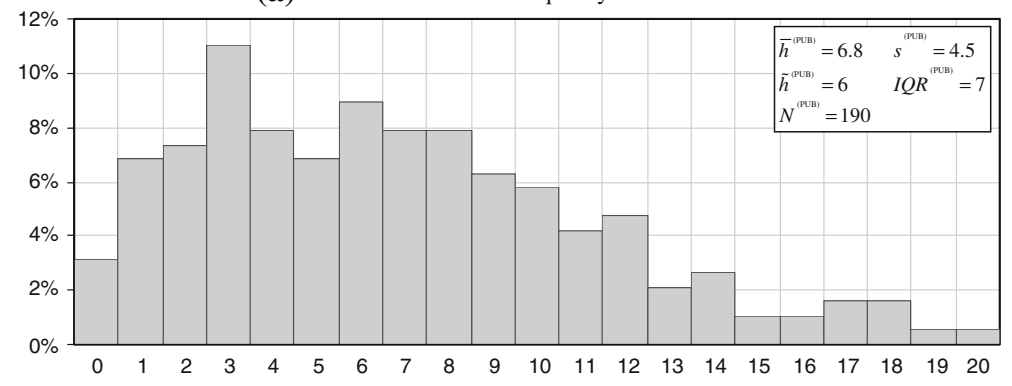

(b) Researchers' relative frequency versus $h^{(\mathrm{PAT})}$-index

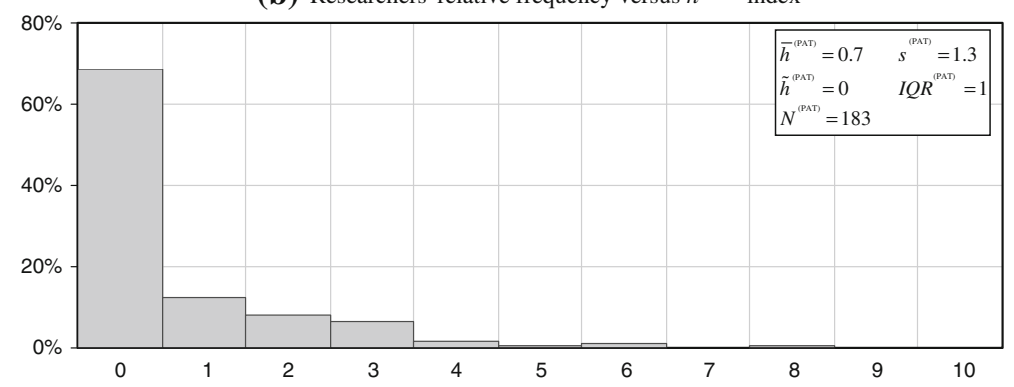

Fig. 2 (Global) $h$-spectra related to the whole set of researchers, respectively for publication (a) and patent analysis (b)

square root of the group size $(\sqrt{N})$. This normalization is quite consistent with other models in the literature, in which the relationship between $h_{\text {GROUP }}$ and $N$ is governed by the power law $h_{\text {GROUP }} \propto N^{\beta}$, with exponent $\beta$ around 0.4-0.5 (Franceschini and Maisano 2011b; Molinari and Molinari 2008).

The advantage of $h_{\mathrm{GROUP}}$, norm with respect to $\bar{h}$ is that it can not be inflated by the co-authorship among members of the same group. For example, in case of systematic co-authorship, the $h$-indices of the individual researchers would artificially increase, with a resulting increase in $\bar{h}$.

$P$ and $C$ are two other indicators influenced by $N$; unsurprisingly, the highest values of these indicators are associated to the group of German researchers. A simple way to enable comparisons among groups on the basis of the members' "average efficiency" is to use the normalised indicators $P / N$ and $C / N$ (see Fig. 3). Analysing these and other indicators that are not influenced by $N$-such as $\bar{h}$ and $h_{\text {GROUP, norm }}$-some interesting results emerge.

Regarding publications, Germans are overcome in terms of impact/diffusion (depicted by $C^{(\mathrm{PUB})} / N^{(\mathrm{PUB})}$ values) by the group of Danish and that of British researchers. This is due to the fact that, on average, publications of DEU are less cited than those of other groups. A confirmation is represented by the relatively small $C P P^{(\mathrm{PUB})}$ and $h_{\mathrm{GROUP}}^{(\mathrm{PUB})}$, norm, with respect to other groups (see Table 2).

Regarding patents, Swiss researchers dominate, since their productivity and impact/ diffusion is much higher than the other researchers', as evidenced by the very high $P^{(\mathrm{PAT})}$ / $N^{(\mathrm{PAT})}, C^{(\mathrm{PAT})} / N^{(\mathrm{PAT})}, h_{\mathrm{GROUP}}^{(\mathrm{PAT})}$ norm and $C P P^{(\mathrm{PAT})}$ values (see Fig. 3 and Table 2). Conversely, Italian researchers show a very low propensity to patent $\left(\% P_{0}{ }^{(\mathrm{PAT})}=89 \%\right)$. 
(a) Publication analysis

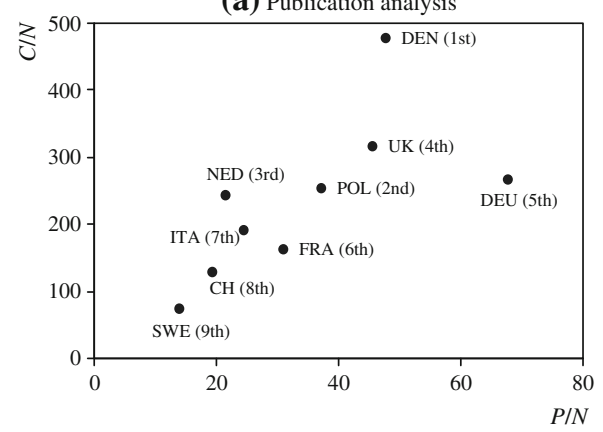

(b) Patent analysis

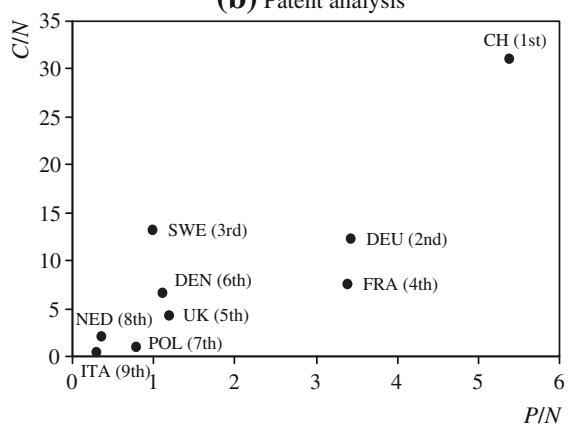

Fig. $3 C / N$ versus $P / N$ for the groups of researchers from the same country, both at publication (a) and patent level (b). Numeric values are reported in Table 2. In brackets are reported the ranks obtained on the basis of the groups' $h_{\mathrm{GROUP}}$, norm value, which aggregates the information relating to publications/patents of a group and corresponding citations (see Table 2)

Table 3 Comparison among academic and non-academic researchers with respect to their propensity to publish or patent

\begin{tabular}{|c|c|c|c|c|c|c|c|c|}
\hline \multirow[t]{2}{*}{ Affiliation type } & \multicolumn{4}{|c|}{ Publications } & \multicolumn{4}{|l|}{ Patents } \\
\hline & Mean $P$ & Mean $C$ & Mean $C P P$ & $\% P_{0}$ & Mean $P$ & Mean $C$ & Mean $C P P$ & $\% P_{0}$ \\
\hline Academic & 49.1 & 259.0 & 5.3 & 0.6 & 2.0 & 8.6 & 4.3 & 62.7 \\
\hline Non-academic & 32.7 & 167.0 & 5.1 & 0.0 & 3.4 & 8.8 & 2.6 & 46.7 \\
\hline
\end{tabular}

For each of the two categories of researchers, the following indicators are reported: mean total publications/ patents per-capita (mean $P$ ), mean total citations per-capita (mean $C$ ), mean $C P P$ and percentage of researchers with no publications or patents $\left(\% P_{0}\right)$

A number of issues, that deserve further study, arise from these specific considerations:

- The different trend in publishing and patenting is the result of a conscious decision by researchers?

- Are there any external influences in the publishing/patenting behaviour, such as government regulations or (dis)incentives?

- Researchers with poor patent output are really unable to realize technology transfer?

These questions have been abundantly discussed in the literature (Van Looy et al. 2006; Mattson et al. 2008; Wong and Singh 2010), although not specifically within the scientific field of interest. Probably a combination from the above factors contributes to generate the observed differences.

Finally, the groups' $h_{2}$ values are reported in Table 2. Two problems can arise with this indicator: (1) it is influenced by $N$ and (2) it is low discerning when $N$ values are quite small. Generally, the synthesis provided by $h_{2}$ becomes relevant when the number of the group members and the corresponding $h$-values have roughly the same order of magnitude, so-despite their different nature-they can be compared (Franceschini and Maisano 2010a). For this reason, in case of patents, we note that $h_{2}$ is not as discriminatory as in the case of publications. 


\section{Publishing and patenting: any relationship?}

The most interesting aspect that emerges when comparing results of the publication and patent analysis, is the lack of correlation between these two kinds of research output. Precisely, there is no correlation $\left(R^{2} \approx 0\right)$ between the $P^{(\mathrm{PAT})}$ and $P^{(\mathrm{PUB})}$ values of individual researchers. We are aware that, in other scientific fields, it was found a general positive relation, supporting the thesis that these activities may actually reinforce one another (Van Looy et al. 2006; Breschi and Catalini 2010; Wong and Singh 2010).

Also, we analysed possible differences between academic and non-academic researchers. Although there is no apparent correlation among publication and patent productivity, there are some differences in terms of average amount of production. Precisely, the average total production of publications per capita (represented by the mean $P^{(\mathrm{PUB})}$ in Table 3) of academics is higher than that one for non-academics. This means that academics tend to be more inclined to publish, even if-regarding the average impact/ diffusion (represented by the mean $C P P$ ) — the difference is very little. As regards patents, we notice the opposite situation: productivity (represented by the mean $P^{(\mathrm{PAT})}$ in Table 3) of non-academics is generally higher than that of academics. This is also confirmed by the high percentage of academics with no patents $\left(\% P_{0}^{(\mathrm{PAT})}\right)$. Regarding the mean $C P P^{(\mathrm{PAT})}$, academics are predominant. However, this rather surprising result is given by the fact that, mean $C P P^{(\mathrm{PAT})}$ of academics is strongly influenced by the contribution of two researchers, with astonishingly high $C$ values.

\section{Aggregation of the two analysis perspectives}

Researchers have been analysed from the two (separate) perspectives of publications and patents. Their aggregation remains an open issue, albeit it can be partially overcome by introducing some maps, which depict the research output positioning of the researchers on the basis of two indicators associated to the perspectives of interest. For example, the map

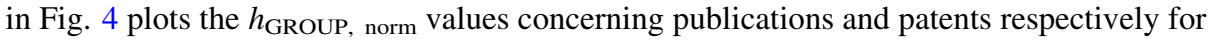
each of the nine groups of researchers. It can be noticed an apparent lack of correlation

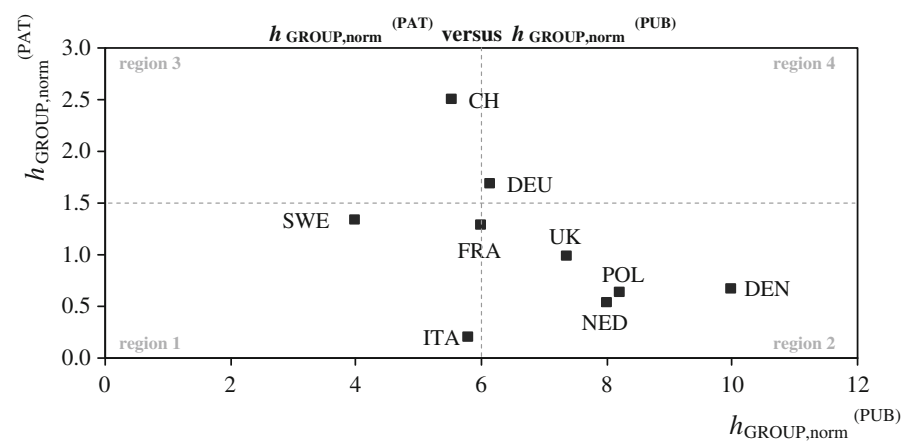

Fig. 4 Map illustrating the relationship between the $h_{\mathrm{GROUP}}^{\text {(PAT) norm }}$ and $h_{\mathrm{GROUP}}^{(\mathrm{PUB})}$ norm for groups of researchers from the same country. The map makes it possible to (qualitatively) identify different regions: 1 groups with relatively low performance in terms of patents and publications; 2 groups relatively efficient in terms of publications but not in terms of patents; 3 groups with medium-high performance in terms of patents but relatively poor performance in terms of publications, and 4 groups with a remarkable performance both in terms of publications and patents 
between the indicators of interest, confirming that-in the discipline concerned-publishing and patenting are quite independent activities.

\section{Concluding remarks}

The proposed analysis is based on a limited sample of researchers, thus it is wild to extend the results associated to national groups to the whole national communities of researchers in the field of Production Technology and Manufacturing Systems.

Another limitation is that - being based on $h$-index-most of the indicators in use could be subjected to the benefits but also criticisms made to $h$-index itself (e.g., they are sensitive to co-authorship, age of publications/patents, type of publications/patent, self citations, seniority of scholars, etc. (Franceschini and Maisano 2010a).

Nevertheless, the fact remains that this work has provided some remarkable initial results on the propensity to publishing and patenting of European researchers in the discipline of interest. Regarding the future, some cues for future research are (1) extending the study to a larger sample (both in terms of researchers and examined countries); (2) studying the time evolution of the attitude to patent/publish by researchers from different countries; (3) investigating the predominant types of technologies that are being used in each country and whether they are worthwhile to the industrial community; (4) studying the possible correlation between the number of publications/patents of a group of researchers and the relevant funding.

\section{References}

Agrawal, A., \& Henderson, R. (2002). Putting patents in context: Exploring knowledge transfer from MIT. Management Science-Special Issue on University Entrepreneurship and Technology Transfer, 48(1), 44-60.

Bar-Ilan, J. (2010). Citations to the 'introduction to informetrics' indexed by WOS, Scopus and Google Scholar. Scientometrics, 82(3), 495-506.

Bornmann, L., Mutz, R., Neuhaus, C., \& Daniel, H. D. (2008). Citation counts for research evaluation: Standards of good practice for analyzing bibliometric data and presenting and interpreting results. Ethics in Science and Environmental Politics, 8(1), 93-102.

Breschi, S., \& Catalini, C. (2010). Tracing the links between science and technology: An exploratory analysis of scientists' and inventors' networks. Research Policy, 39(1), 14-26.

Calderini, M., Franzoni, C., \& Vezzulli, A. (2009). The unequal benefits of academic patenting for science and engineering research. IEEE Transactions on Engineering Management, 56(1), 16-30.

Cheng, Y. H., Kuan, F. Y., \& Chuang, S. C. (2010). Profitability decided by patent quality? An empirical study of the U.S. semiconductor industry. Scientometrics, 82(1), 175-183.

CIRP. (2011). Organization website. Retrieved 10 April, 2011 from http://www.cirp.net.

Criscuolo, P., \& Verspagen, B. (2008). Does it matter where patent citations come from? Inventor vs. examiner citations in European patents. Research Policy, 37(10), 1892-1908.

Czarnitzki, D., Glänzel, W., \& Hussinger, K. (2007). Patent and publication activities of german professors: An empirical assessment of their co-activity. Research Evaluation, 16(4), 311-319.

Egghe, L. (2010). The Hirsch-index and related impact measures. In B. Cronin (Ed), Annual Review of Information Science and Technology (ARIST) (Vol. 44). ISBN 978-1-57387-371-0.

Franceschini, F., Galetto, M., \& Maisano, D. (2007). Management by measurement: Designing key indicators and performance measurement systems. Berlin: Springer Verlag.

Franceschini, F., \& Maisano, D. (2010a). Analysis of the Hirsch index's operational properties. European Journal of Operational Research, 203(2), 494-504.

Franceschini, F., \& Maisano, D. (2010b). The Hirsch spectrum: A novel tool for analysing scientific journals. Journal of Informetrics, 4(1), 64-73. 
Franceschini, F., \& Maisano, D. (2011a). Bibliometric positioning of scientific manufacturing journals: A comparative analysis. Scientometrics, 86(2), 463-485.

Franceschini, F., \& Maisano, D. (2011b). Structured evaluation of the scientific output of academic research groups by recent $h$-based indicators. Journal of Informetrics, 5(1), 64-74.

Geuna, A., \& Nesta, L. J. J. (2006). University patenting and its effects on academic research: The emerging European evidence. Research Policy, 35(6), 790-807.

Guan, J. C., \& Gao, X. (2009). Exploring the $h$-index at patent level. Journal of the American Society for Information Science and Technology, 60(1), 35-40.

Guan, J., \& He, Y. (2007). Patent-bibliometric analysis on the Chinese science-Technology linkages. Scientometrics, 72(3), 403-425.

Hirsch, J.E. (2005). An index to quantify an individual's scientific research output. Proceedings of the National Academy of Sciences of the United States of America (Vol. 102, pp. 16569-16572.

Labbé, C. (2010). Ike Antkare one of the great stars in the scientific firmament. ISSI Newsletter, 6(2), 48-52.

Lazaridis, T. (2010). Ranking university departments using the mean h-index. Scientometrics, 82(2), 211-216.

Mattson, P., Laget, P., Nilsson, A., \& Sundberg, C. J. (2008). Intra-EU vs. extra-EU scientific co-publication patterns in EU. Scientometrics, 75(3), 555-574.

Molinari, A., \& Molinari, J. F. (2008). A new methodology for ranking scientific institutions. Scientometrics, 75(1), 163-174.

Nagpaul, P. S., \& Roy, S. (2003). Constructing multi-objective measure of research performance. Scientometrics, 56(3), 383-402.

Rousseau, R. (2006) New developments related to the Hirsch index. E-prints in Library and Information Science (ELIS). Retrieved April 10, 2011 www.eprints.rclis.org.

Schubert, A. (2007). Successive $h$-indices. Scientometrics, 70(1), 201-205.

Scopus-Elsevier. (2011). Retrieved April 10, 2011 from www.info.scopus.com.

Shelton, R.D. \& Leydesdorff, L. (2011) Publish or patent: Bibliometric evidence for empirical trade-offs in national funding strategies. 13th Conference of International Society for Scientometrics and Informetrics, Durban, July 4-8.

van Looy, B., Callaert, J., \& Debackere, K. (2006). Publication and patent behavior of academic researchers: Conflicting, reinforcing or merely co-existing? Research Policy, 35(4), 596-608.

Wong, P. K., \& Singh, A. (2010). University patenting activities and their link to the quantity and quality of scientific publications. Scientometrics, 83(1), 271-294. 